\title{
SUDDEN DEATH IN SPORT
}

\author{
W S Hillis, P D McIntyre, J Maclean, J F Goodwin, W J McKenna
}

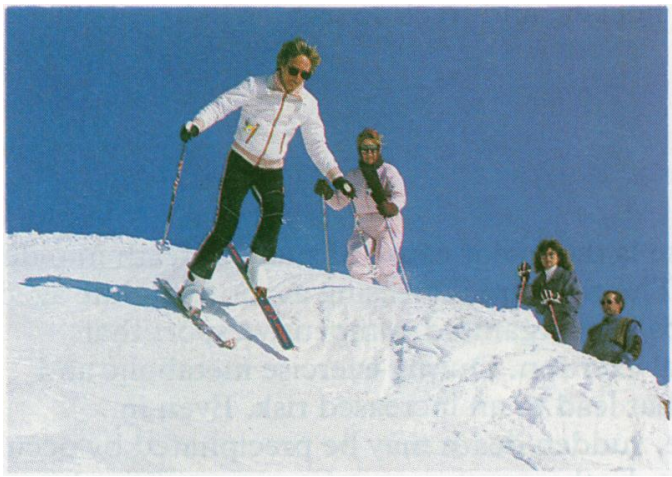

Skiing is a high intensity sport that makes moderate or high dynamic and static demands.

\section{Cardiac causes of sudden death}

Coronary artery disease

Hypertrophic cardiomyopathy

Idiopathic concentric left ventricular hypertrophy

Congenital anomalies of coronary arteries

Aortic rupture

Right ventricular dysplasia

Myocarditis

Valvular disease

Arrhythmias and conduction defects

Congenital heart disease, operated or unoperated
Most sudden deaths in sport are caused by cardiovascular conditions. The cardiovascular benefits of exercise are well established, and epidemiology studies suggest that long term exercise programmes may reduce the risk of sudden death. Increasing leisure time and facilities promote sports participation at all ages. A few people are at risk of serious arrhythmia or sudden death with exercise. The cause of death varies with the age of participants; congenital structural abnormalities occur in younger age groups and coronary artery disease in older age groups. Identifying such abnormalities makes prevention possible.

Sudden death in sport is uncommon, with an incidence of 2 cases per 100000 subject years. Five in 100000 athletes have a condition that might predispose them to serious cardiac problems, and of those at risk $10 \%$ ( 1 in 200000 ) may die suddenly or unexpectedly.

Considerable controversy exists about the cost effectiveness of screening all young people by examination before participation. Alternative views suggest targeting people with a family history of sudden death or premature coronary artery disease and educating all participants to see their doctors about even minor warning symptoms.

Some of the cardiac causes of sudden death will be suggested by symptoms and signs on clinical examination. The importance of others may be established only by detailed cardiological investigations including electrocardiography, chest radiography, echocardiography, doppler cardiography, and, rarely, cardiac catheterisation. Exercise testing is of major value and 24-48 hour tape electrocardiographic recordings may be required. Potentially serious symptoms such as syncope, pre-syncope, palpitation, chest pain, and undue dyspnoea should stimulate taking a detailed clinical history, making a detailed examination, and referring for cardiological investigation if appropriate. A family history of cardiac abnormality would suggest a hereditary basis.

\section{General considerations}

\section{Classification of sports according to intensity and demands}

$\begin{array}{ll}\text { A High intensity } & \\ 1 \text { Moderate or high dynamic and static demands } \\ \text { American football } & \text { Fencing } \\ \text { Boxing } & \text { Ice hockey } \\ \text { Cross country skiing } & \text { Rowing } \\ \text { Downhill skiing } & \text { Rugby }\end{array}$

2 Moderate to high dynamic, low static demands
Badminton

Baseball

Basketball

Field hockey

Lacrosse
Running (sprinting)

Speed skating

Water polo

Wrestling

Squash

Swimming

Table tennis

Tennis

Volleyball

3 Low dynamic, moderate to high static demands

$\begin{array}{lll}\text { Archery } & \text { Field events } & \text { Motor cycling } \\ \text { Auto racing } & \text { (jumping and } & \text { Sailing } \\ \text { Diving } & \text { throwing) } & \text { Ski jumping } \\ \text { Equestrian events } & \text { Gymnastics } & \text { Water skiing } \\ & \text { Karate or judo } & \text { Weight lifting }\end{array}$

B Low intensity, low dynamic and static demands

$\begin{array}{lll}\begin{array}{l}\text { Bowling } \\ \text { Cricket }\end{array} & \text { Curling } & \text { Golf }\end{array}$

By far the most common condition leading to sudden death during exercise, usually in those aged over 40 , is coronary artery disease, including congenital abnormalities of the coronary artery tree. Death in younger athletes is rare but may be due to hypertrophic cardiomyopathy, right ventricular dysplasia, valvular heart disease such as aortic stenosis, and Marfan's syndrome.

Consensus discussions have suggested classification into high and low intensity sports, with further differentiation into those with major dynamic and static components. 


\section{Classification of sports according to danger of body collision}

\section{Contact sports}

American football

Boxing

Ice hockey

Karate or judo

Lacrosse

Rugby

Soccer

Wrestling

\section{Non-contact sports}

Auto racing Bicycling

Diving

Downhill skiing

Equestrian events

Gymnastics

Motor cycling

Polo
Ski jumping

Water polo

Water skiing

Weight lifting

(increased risk if

syncope occurs)
Contact and non-contact sports must be distinguished and activities identified that may place a person in jeopardy if syncope occurs. Patients with a prosthetic cardiac valve should not participate in contact sports because of potential valve dehiscence during forceful chest contact and the risks of trauma in the presence of anticoagulant drugs. Most cardiovascular risk occurs with extreme exertion, such as marathon running, cross country running, skiing, basketball, football, hockey, and track sports.

\section{Coronary artery disease}

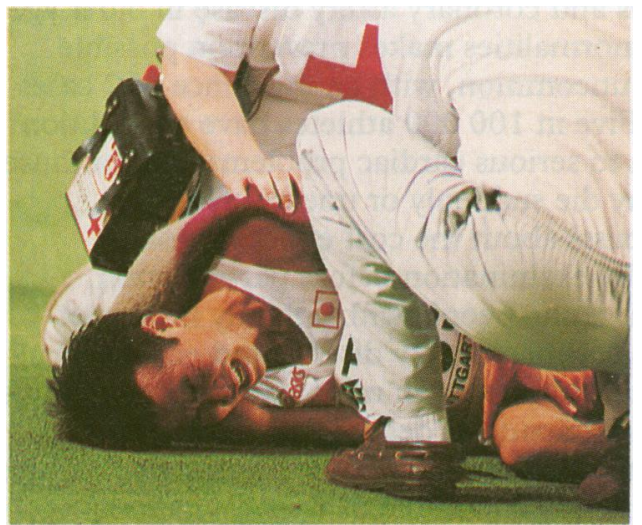

Marathon running is one of the most common causes of sudden death from coronary artery disease.



During 24-48 hour tape electrocardiography the contacts and leads are covered by clothing and the recorder fixed to the belt.



Exercise testing is useful to diagnose many cardiac disorders.
Coronary artery disease is the major cause of sudden death in older athletes (aged over 35 ). The risks are increasing as more middle aged and older people participate in organised competitive sport that requires vigorous physical exertion. During exercise metabolic and physical changes occur that lead to an increased risk. Even in conditioned older people, sudden death may be precipitated by occult coronary atheroma. Most deaths occur in running, competitive long distance racing, jogging, and other vigorous sports such as rugby, soccer, and squash.

\section{History and risk factors}

Previous symptoms suggestive of coronary atherosclerosis have often been recognised, and risk factors are frequently present, including smoking, a family history of myocardial infarction at under 55 years, hypertension, and hypercholesterolaemia. The victims are often perceived as being very fit and may have competitive personalities.

\section{Pathology}

In pathological studies, obstructive atheromatous coronary artery lesions are usually found, often associated with thrombus. The myocardium may show previous unrecognised healed infarcts.

\section{Prevention}

Extreme forms of conditioning, including marathon running, do not prevent severe atherosclerosis or sudden death. Education should be emphasised to increase awareness of warning symptoms such as chest pain, palpitation, or syncope. Severe exercise should be undertaken cautiously in patients aged over 40 , particularly those with risk factors.

\section{Diagnosis}

The use of electrocardiograph stress testing to detect those at risk may have significant limitations. In addition, the incidence of false positives may be as high as $25 \%$ in sportsmen. People should be assessed in terms of their functional capacity and risk factors.

\section{Degree of risk}

Subjects with known coronary artery disease can be classified as being at low, moderate, or high risk by assessing left ventricular function, screening for evidence of reversible ischaemia, and comparing with the normal exercise capacity for age. Exercise testing may also detect ventricular arrhythmias or a reduced blood pressure response. High risk patients are those with decreased left ventricular systolic function at rest. Moderate risk patients are those with reduced exercise capacity for age, evidence of reversible ischaemia, ventricular tachycardia, or reduced systolic blood pressure. Low risk subjects with normal left ventricular function or without reversible ischaemia can participate in low intensity competitive sports, but those with moderate or high risk should be excluded. In patients who have had coronary artery bypass grafting or angioplasty serial assessment after the procedure should be undertaken. 


\section{Prognostic factors for sudden death caused by hypertrophic cardiomyopathy}

- Family history of sudden death

- Documented ventricular tachycardia

- Young age at onset of symptoms

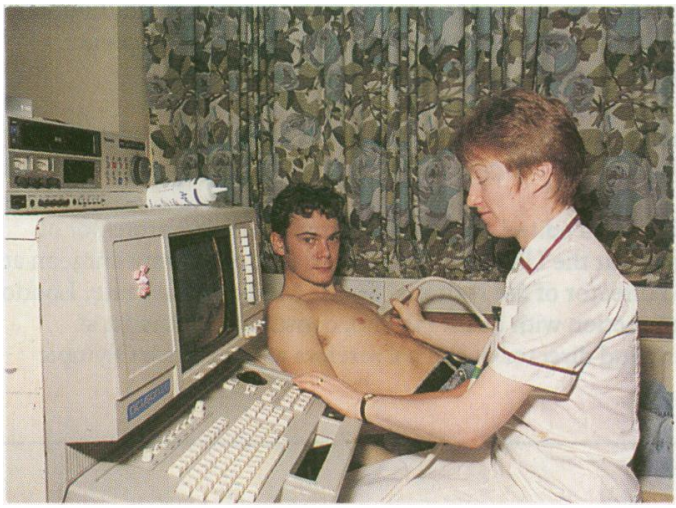

Echocardiography is the diagnostic test for " hypertrophic cardiomyopathy.

\section{Other causes}

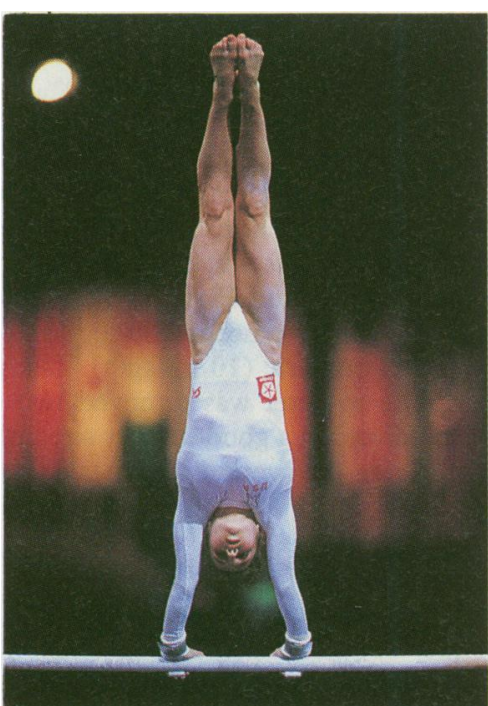

Gymnastics is a high intensity sport that makes low dynamic and moderate to high static demands.
Hypertrophic cardiomyopathy is the leading cause of sudden death in young athletes.

\section{Anatomical abnormalities}

A hypertrophied, non-dilated left ventricle exists but no history of predisposing diseases. The left ventricular chamber size is reduced and the muscular hypertrophy impairs diastolic filling. Left ventricular outflow tract obstruction may be secondary to hypertrophy of the subaortic septum or to systolic anterior motion of the mitral valve. There are predispositions to supraventricular and ventricular arrhythmias; ventricular tachycardia may lead to ventricular fibrillation.

\section{Cause}

Hypertrophic cardiomyopathy is inherited as an autosomal dominant condition that has a high degree of penetrance. When it is identified in an individual screening of all first degree relatives should be considered.

\section{Symptoms}

Patients may present with chest pain, palpitation, syncope, or breathlessness or may be asymptomatic. Abnormal cardiac findings may consist of a jerky or bisferiens pulse or an increased or double left ventricular apical impulse with a palpable fourth heart sound and a systolic murmur at the lower left sternal border that is decreased by squatting and increased by standing. The electrocardiogram is usually abnormal, although the findings may be non-specific. Cardiomegaly may be present on chest radiography but the diagnosis is made on echocardiography, which shows a small cavity and hypertrophy of the left ventricle, limited compliance, and limited diastolic filling.

\section{Future exercise}

The presence of cardiomyopathy should restrict people from engaging in strenuous dynamic or static sports, but those at low risk may follow a moderate exercise programme.

\section{Differentiation from "athletic heart}

Left ventricular hypertrophy in athletes is common as a normal response to training, but the ventricular wall is rarely more than $12 \mathrm{~mm}$ thick. A thickness of more than $16 \mathrm{~mm}$ is strongly suggestive of hypertrophic cardiomyopathy.

\section{Right ventricular dysplasia}

Right ventricular cardiomyopathy or dysplasia has been reported as a cause of sudden death during sport, and may have a regional distribution. It is associated with fibrous, fatty replacement of the right ventricular myocardium, which is grossly dilated.

\section{Congenital coronary artery abnormalities}

Patients may present with symptoms of anginal pain, syncope with exertion, or even sudden death. Investigations should include a 12-lead electrocardiogram, a two dimensional echocardiogram to assess the position of origin of the left main coronary artery, and an exercise stress test.

\section{Marfan's syndrome}

Cardiovascular involvement gives mitral valve prolapse in almost all patients. If the syndrome is suspected on clinical appearance and by the presence of a family history the patient should be referred for regular echocardiography. $\beta$ blockers should be considered to retard the rate of aortic root dilatation and may be prescribed for patients with dilated aortic roots, and for those who have had aortic surgery.

\section{Aortic stenosis}

Symptoms from aortic stenosis of left ventricular failure, syncope, or anginal pain occur late. In those with a symptomatic, mild to moderate stenosis, participation may occur in low intensity sports (class B) or alternatively moderate static or dynamic demands (class A3). 




Golf is a low intensity sport with little danger of body collision.

\section{Issues requiring further study include:}

- Identifying causes of sudden death during physical exercise

- The appropriateness, cost, and practicality of cardiovascular screening of presumably healthy children and adolescents before they participate in sport and of other people before they continue or return to sport

- Counselling patients with known cardiac abnormalities about their levels of activity and about the risk and safety of specific sporting events

- Establishing guidelines for disqualification from competition

\section{Mitral valve prolapse}

The approach to mitral valve prolapse should be pragmatic. In the absence of symptoms and signs or associated lesions and with a negative family history, full sporting participation should be allowed. In the presence of palpitations, dizziness, or near syncope, both Holter monitoring and stress testing are invaluable for estimation of the mechanism of the symptoms. Associated problems that might exclude sporting activity include an associated prolonged QT syndrome or a family history of sudden death associated with a mitral valve prolapse.

\section{Valve replacement}

In patients who have had valvuloplasty or annuloplasty, contact sports should be avoided. Athletes with a prosthetic valve and normal cardiac function may engage in low intensity sports.

W S Hillis is reader and consultant cardiologist, P D McIntyre is career registrar, and $\mathrm{J}$ Maclean is research fellow in the department of medicine and therapeutics of the University of Glasgow at the Gardiner Institute, Western Infirmary, Glasgow. J F Goodwin is emeritus professor of cardiology at the Royal Postgraduate Medical School, Hammersmith, London. W J McKenna is professor of cardiology in the department of cardiological sciences at St Georges Hospital Medical School, London.

The pictures of the skier, marathon runner, gymnast, and golfer are reproduced with permission from Spectrum Colour Library, Allsport, Vandystadt, and Colorsport.

The ABC of Sports Medicine has been edited by Greg McLatchie, visiting professor of sports medicine and surgical sciences at the University of Sunderland, consultant surgeon at Hartlepool General Hospital, and director of the National Sports Medicine Institute, London. This paper has been edited in conjunction with Mark Harries, consultant physician at Northwick Park Hospital, Harrow, and director of clinical services at the British Olympic Medical Centre, Harrow.

\title{
Lesson of the Week
}

\section{Male ritual circumcision resulting in acute renal failure}

\author{
Julian D Eason, Maria McDonnell, Godfrey Clark
}

\section{Tight bandaging after male ritual circumcision may cause urinary obstruction and acute renal failure. Improved community education reduces the risks}

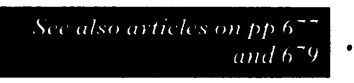

Department of Paediatric Nephrology and Optimum Health Services, Guy's Hospital, London SE1 9RT Julian D Eason, paediatric registrar

Maria McDonnell, senior community medical officer Godfrey Clark, consultant paediatric nephrologist

Correspondence to: Dr Clark.
Male ritual circumcision is commonplace in the British community, especially among those of Jewish and Muslim faiths and certain immigrant populations such as West Africans. We report three cases of circumcision performed in the community in which the parents were not warned about complications and which resulted in prolonged urinary retention and acute renal failure.

\section{Case reports}

CASE 1

A 15 day old baby was admitted with a four day history of increasing vomiting and abdominal distension. After a normal pregnancy and birth he had been admitted to this hospital's special care baby unit for one day because of tachypnoea, grunting, and poor feeding. He had been circumcised by a local community rabbi at 11 days of age. Increasing vomiting and abdominal distension were subsequently noted, but he continued to feed well. His mother thought that he was passing urine normally.

On physical examination he had a pulse rate of 140 beats/min, warm hands and feet, a blood pressure of $77 / 52 \mathrm{~mm} \mathrm{Hg}$, and a respiratory rate of 28 per minute. The bladder was palpable above the umbilicus, but the abdomen wall was soft with normal bowel sounds. His penis was tightly bandaged, and the circumcision wound was inflammed and purulent. Table I shows the results of his initial renal function tests.
After removal of the penile dressing the bladder was catheterised through the urethra and chloramphenicol ointment applied to the wound. A wound swab contained mixed Gram negative organisms on culture. Intravenous cefotaxime was also prescribed. Urine output was replaced hourly with $0.9 \%$ intravenous saline as his initial diuresis after relief of the urinary obstruction was large $(1129 \mathrm{ml})$.

The following day he became irritable with repetitive myoclonic jerking in his arms and legs. Anticonvulsants were given for three days, but no cause for the abnormal movements was found. Plasma electrolyte concentrations had returned to normal by that time (table II), but he was still polyuric $(10 \mathrm{~m} / \mathrm{kg} / \mathrm{h})$. The jerking did not recur. The catheter was removed two days later, after which he voided a good urinary stream. At review one month later the wound had healed and he was thriving.

\section{CASE 2}

A male baby of Nigerian parents was born at this hospital at term after a normal pregnancy. At the age of 1 month he was circumcised by a non-medical community practitioner at home. Two days later he began screaming and stopped feeding, be came jittery, and his parents noticed that his abdomen was distended; when seen in the accident and emergency department he had not passed urine for at least 16 hours. On physical examination his pulse rate was 180 beats $/ \mathrm{min}$, 